\title{
ESTIMATING THE ABUNDANCE OF SAMBAR DEER( Cervus unicolor unicolor) AT THE HORTON PLAINS NATIONAL PARK OF SRI LANKA
}

\author{
I.K.Rajapakshe', U.K.G.K. Padmalal', S.W. Kotagama² \& N.K. Athulathmudali \\ 'Department of Zoology, The Open University of Sri Lanka \\ 2Department of Zoology, University of Colombo \\ ${ }^{1}$ National Zoological Garden, Dehiwala
}

A study was conducted during the period 1997-2000 to estimate the sambar population at the Horton Plains National Park of Sri Lanka using direct and indirect method. Vehicle based spot light technique was used as the direct method and fecal accumulation method was used as the indirect method. The defecation rate of sambar used in the indirect method was estimated by, a study carried out in the National Zoological Garden Dehiwala, using eight-penned sambar. The mean defecation rate of sambar deer for the eight focal sampling at the Zoological Garden - Dehiwala was twenty-one pellet groups / sambar/ day (range = 20,17-30). Results of ANOVA revealcd that there is a significant variation in defecation rate with age $(P<0.05$, Sig-0.02) but it is not significant sex $(P<0.05$, Sig-0.09).

Altogether 688 transects were run for the direct method. For the indirect method, 200 quadrats measuring $25 \mathrm{~m} * 25 \mathrm{~m}$ size were sampled. Standard equations were used for the population analysis. The estimated sambar population, crude density and ecological density of the direct and indirect methods are $2102.5=\mathrm{B} 1845,66.5 / \mathrm{km}^{2}, 223.5 / \mathrm{km}^{2}$ and $3154=\mathrm{B} 11096,98.9 \mathrm{~km}^{2}, 350 / \mathrm{km}^{2}$ respectively.

Results indicated that the values obtained by the indirect method are higher than the values obtained from the direct method. The reliability of the indirect method depends on the actual daily defecation rate of sambar deer, which depends on the habitat use. Direct methods are more reliable but not feasible at all times. However indirect method can be used to obtain information on population trends. The results of this study indicate an increase in sambar deer population at the Horton Plains National Park when compared with estimates made in 1991.

Proceedings of the Eighth Annual Forestry and Environment Symposium 2002 of the Department of Forestry and Environmental Science, University of Sri Jayewardenepura. Sri Lanka 